Review

\title{
Epidemiology, Risk Factors and Measures for Preventing Drowning in Africa: A Systematic Review
}

\author{
Lauren Miller $^{1}{ }^{(\mathbb{D}}$, Faith O. Alele ${ }^{1,2, * \mathbb{C}}$, Theophilus I. Emeto ${ }^{1}\left(\mathbb{D}\right.$ and Richard C. Franklin ${ }^{1} \mathbb{C}$ \\ 1 Public Health and Tropical Medicine, College of Public Health, Medical and Veterinary Sciences, \\ James Cook University, Townsville, QLD 4811, Australia; lauren.miller4@my.jcu.edu.au (L.M.); \\ theophilus.emeto@jcu.edu.au (T.I.E.); richard.franklin@jcu.edu.au (R.C.F.) \\ 2 College of Healthcare Sciences, James Cook University, Townsville, QLD 4811, Australia \\ * Correspondence: faith.alele@my.jcu.edu.au; Tel.: +61-7-4781-5082
}

Received: 27 June 2019; Accepted: 18 September 2019; Published: 25 September 2019

\begin{abstract}
Background and Objectives: Drowning is a leading cause of unintentional injury related mortality worldwide, and accounts for roughly 320,000 deaths yearly. Over $90 \%$ of these deaths occur in low- and middle-income countries with inadequate prevention measures. The highest rates of drowning are observed in Africa. The aim of this review is to describe the epidemiology of drowning and identify the risk factors and strategies for prevention of drowning in Africa. Materials and Methods: A review of multiple databases (MEDLINE, CINAHL, PsycINFO, Scopus and Emcare) was conducted from inception of the databases to the 1st of April 2019 to identify studies investigating drowning in Africa. The preferred reporting items for systematic review and meta-analysis (PRISMA) was utilised. Results: Forty-two articles from 15 countries were included. Twelve articles explored drowning, while in 30 articles, drowning was reported as part of a wider study. The data sources were coronial, central registry, hospital record, sea rescue and self-generated data. Measures used to describe drowning were proportions and rates. There was a huge variation in the proportion and incidence rate of drowning reported by the studies included in the review. The potential risk factors for drowning included young age, male gender, ethnicity, alcohol, access to bodies of water, age and carrying capacity of the boat, weather and summer season. No study evaluated prevention strategies, however, strategies proposed were education, increased supervision and community awareness. Conclusions: There is a need to address the high rate of drowning in Africa. Good epidemiological studies across all African countries are needed to describe the patterns of drowning and understand risk factors. Further research is needed to investigate the risk factors and to evaluate prevention strategies.
\end{abstract}

Keywords: drowning; immersion injuries; Africa

\section{Introduction}

The World Health Organization (WHO) defines drowning as "the process of experiencing respiratory impairment from either immersion or submersion in liquid" [1]. Drowning is the third leading cause of unintentional injury related cause of mortality worldwide, accounting for $7 \%$ of all injury related deaths. It is a global under recognized and neglected public health burden that claims the lives of 320,000 people every year [2]. More than $90 \%$ of these deaths occur in low- and middle-income countries with inadequate prevention measures [3]. It is among the ten leading causes of deaths in children and young people in the world with children aged less than five years at increased risk [4]. Between 1990 and 2013, drowning rates declined by $52.2 \%$ globally [5], however, despite this decline, the highest rates of drowning were observed in Africa [3]. 
The African continent is unfortunately plagued with the world's most dramatic public health crises with communicable diseases such as HIV/AIDS, malaria, and lower respiratory tract infections as leading causes of death in the region [6]. In addition, there is an increasing burden of non-communicable (hypertension, diabetes and heart) diseases and injuries [6]. The combined burden of both the communicable, non-communicable diseases and injuries have placed a strain on the already weak health systems in addition to struggling economies in the continent [6]. In spite of injuries been identified as a leading cause of death in Africa [4], this public health threat is yet to receive the desired attention, rather the management and prevention of communicable diseases is still a top priority [6].

Africa has recorded considerable success in reducing childhood deaths related to communicable diseases [6]. However, there is limited statistics and data on drowning related deaths which also contributes to infant mortality rates. Age is a leading risk factor for drowning and commonly occurs among children aged 1-4 years [4]. A recent incident that occurred in a West African country, was the case of a thirteen-month-old child who drowned in his parents' indoor swimming pool [7]. This is a common occurrence in low- and middle-income countries (LMICs) and within the region [8]. Unfortunately, unlike high-income countries such as Australia and the United States of America (USA), drowning cases and deaths are under-reported in the African continent [4]. Other risk factors of drowning include, male gender, increased access to water, flooding disasters, commuting on water, lack of supervision and recreational drug use $[4,9,10]$.

The knowledge of the epidemiology and risk factors of drowning aids the development and implementation of policies and strategies that reduce the incidence of drowning. Studies originating from high-income countries like Australia and USA suggest a range of primary and secondary prevention strategies to curb drowning deaths [11-15]. Recommendations proposed include increasing supervision, erecting pool fences, increasing public awareness and education through health promotion and public health advocacy [11-15]. However, these interventions may not be applicable in a region like Africa due to the diversity and variation in the epidemiologic, demographic and cultural factors [8].

Currently, there is no systematic review investigating drowning in Africa. Only three recent reviews on drowning in low- and middle-income countries, drowning in South Africa and Tanzania respectively have been published $[8,16,17]$. Therefore, it is imperative to understand the epidemiology, risk factors and current prevention strategies in Africa to direct policies for the prevention of drowning in Africa. Hence, the aim of this systematic review was to describe the epidemiology of drowning in Africa and to identify the risk factors and proposed and current strategies to prevent drowning.

\section{Methods}

\subsection{Literature Search}

The systematic review was conducted in accordance to the preferred reporting items for systematic review and meta-analysis guidelines (PRISMA) [18]. The PRISMA flow chart for the review is shown in Supplementary Figure S1. A literature search was conducted using Ovid Medline, Emcare, Cumulative Index to Nursing and Allied Health (CINAHL), PsycINFO, and Scopus for original research articles published in English from inception until the 30th of November 2018. The search was updated on the 1st April 2019. We included all articles focusing on drowning in Africa. There were slight variations in the search terms depending on the database. Search terms involved a combination of free text words and Medical Subject Headings (MeSH) terms. General search terms were "drown" and "Africa". The search strategy for Medline is shown in Supplementary Table S1. The study protocol was registered in PROSPERO with registration number CRD42019092758.

\subsection{Eligibility Criteria}

The studies included in this review are published original research reporting drowning in African countries. We applied no limits to the year of publication and included all age groups. In addition, we included studies that reported drowning as part of other injuries studies to capture all data from 
the region. Studies excluded were review articles, drowning because of suicide or homicide, non-fatal drowning or near drowning or hospitalization due to drowning or where fatal drowning could not be distinguished from non-fatal drowning.

\subsection{Data Extraction}

Faith O. Alele (F.O.A.) and Theophilus I. Emeto (T.I.E.) identified all included studies from the search strategy. Uncertainties about the included studies was discussed until consensus was reached. FOA and TIE extracted general and study specific characteristics from the included studies and Lauren Miller (L.M.) and Richard C. Franklin (R.C.F.) crosschecked the data.

\subsection{Quality of Methods Assessment}

The methodological quality of the included studies was assessed by FOA and TIE using the modified quality assessment tool for studies with diverse designs (QATSDD) critical appraisal tool [19]. The tool assesses the validity, reliability and generalizability of studies. The included studies were a mix of cross-sectional, descriptive and case-control studies and each study design were assessed using the appraisal tool. The tool was modified to exclude two items that were not applicable to the included studies. The excluded items comprised of statistical assessment of reliability and validity of measurement tool(s) (Quantitative only), fit between stated research question and format and content of data collection tool e.g., interview schedule (Qualitative), assessment of reliability of analytical process (Qualitative only) and evidence of user involvement in design. In the modified QATSDD tool each criterion was awarded a score of 0 to 3 with $0=$ not at all, $1=$ very slightly, $2=$ moderately and 3 = complete. The scores of the criteria were summed up to assess the methodological quality of included studies with a maximum score of 36. For ease of interpretation, the scores were converted to percentages and were categorised as excellent $(>80 \%)$, good $(50-80 \%)$ and low $(<50 \%)$ quality of evidence based on the overall score (Supplementary Table S2).

\subsection{Data Synthesis}

Drowning was reported exclusively or as part of a wider study such as injury studies. Approximately 28\% (11) of the included studies reported drowning as unintentional. In studies where drowning was unspecified, we reported the drowning as intentional. Measures used to report drowning were proportions and incidence rates. The incidence rates and proportions of drowning were reported using frequency tables. The risk factors for drowning were identified in two articles, one of which only reported drowning as part of a wider study. Therefore, the risk factors identified were extrapolated to drowning. However, given the paucity of information on risk factors associated with drowning, we identified the potential risk factors based on previously identified factors documented in the literature $[3,4,8]$ and based on the reported rates of the potential factors. A meta-analysis was not conducted due to the heterogeneity of the included studies.

\section{Results}

\subsection{Epidemiology of Drowning in Africa}

The included studies were conducted across 15 countries in Africa (Figure 1). The databases searches identified 345 articles, of which 42 articles were included in the review after screening for titles, abstracts and full text review (Supplementary Figure S1). Three (3) studies reported drowning in multiple sites (countries) [20-22]. Twenty-four (57\%) of the articles originated from South Africa [20-43], three (7.1\%) were from Ethiopia [20,44,45], Ghana [20,46,47], Malawi [20,48,49], Nigeria [50-52] and Uganda [53-55] respectively, while 2 (4.8\%) studies were from Cote d'Ivoire [20,56], Kenya [20,57] and Egypt [21,22] respectively. One (2.4\%) article each originated from Burkina Faso [20], Guinea [58], The Gambia [20], Tanzania [59], Seychelles [60] and Zimbabwe [61], See Figure 1 for location. The most commonly used data were surveillance data (46\%) and death registers including 
hospital, police and coronial reports. Twelve (12) studies investigated and described drowning exclusively [21-27,30,43,50,54,60], while in 30 studies, drowning was reported as part of a wider study including studies investigation all cause of death and external causes of death $[20,28,29,31-42,44-49$, $51-53,55-59,61]$. Measures used to describe drowning were proportions and rates. Drowning rates in children were reported in thirteen (13) studies [21,28,30,31,33,35,37-39,42,46,49,52], while $29(69 \%)$ studies described drowning rates of adults and children or adults alone $[20,22-27,29,32,34,36,40,41$, $43-45,47,48,50,51,53-61]$. The denominators used varied by country. In some studies, the estimated proportion or rate of drowning was based on the total population in the study area. By contrast, other studies reported the mortality rates for external causes and all cause of deaths. All deaths due to injuries, trauma and external causes were considered as mortality due to external causes using the ICD 11 classification [62].

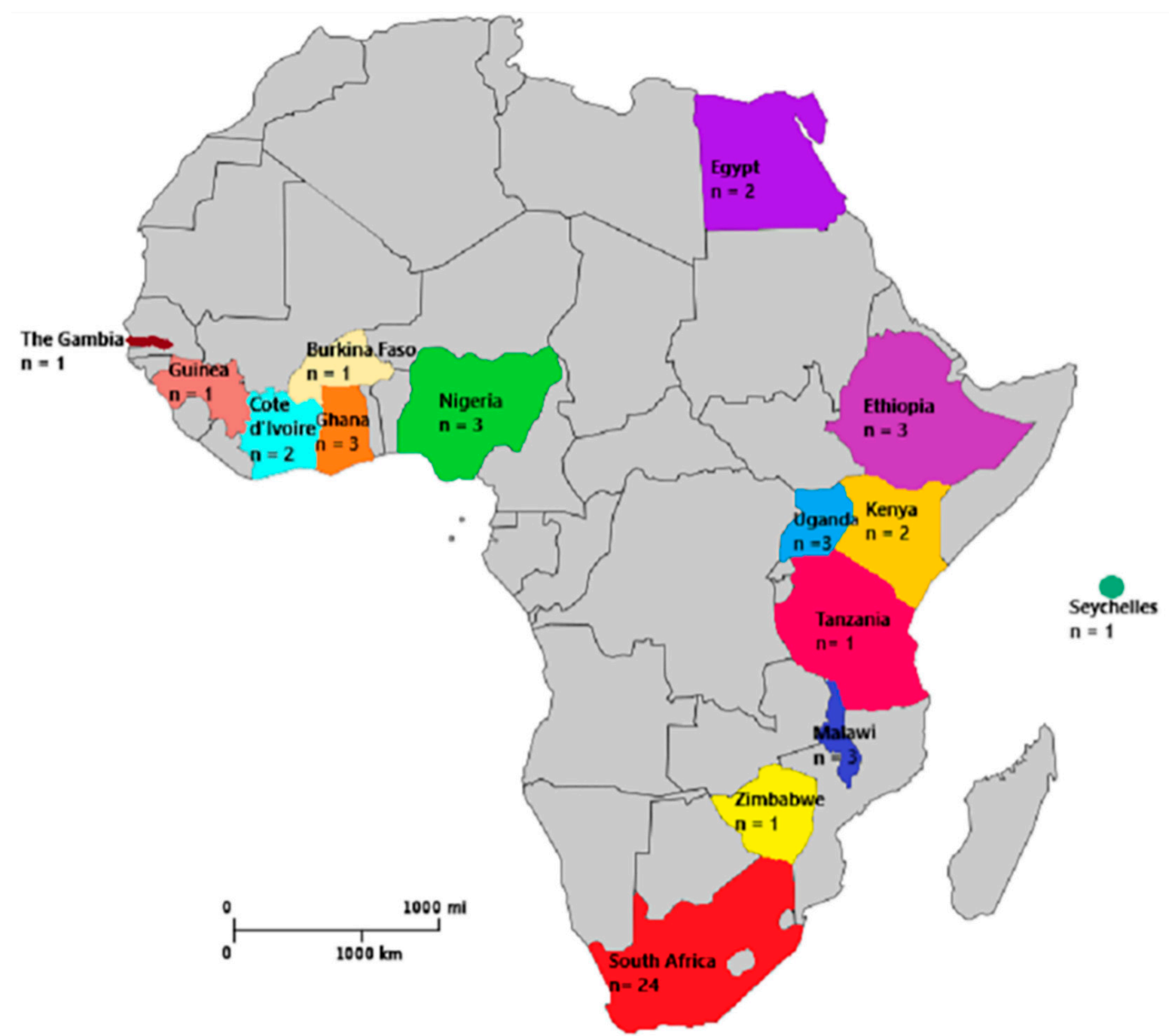

Figure 1. Map of Africa showing the countries and number of studies originating from each country was modified from Wikimedia Commons [63].

\subsection{Drowning Rates in Africa}

In Table 1, twelve (12) investigated drowning exclusively in different regions of Africa [21-27, $30,43,50,54,60]$. However, there were variable methods of reporting drowning across the different studies. Among population-based studies, the proportion of drowning fatalities ranged from $0.019 \%$ to $1.2 \%[23,26]$. In studies where all submersion events were reported, unintentional drowning accounted 
for $80 \%$ of drowning deaths in one study [50], while accidental drowning accounted for $10.7 \%$ of all submersion (near drowning and drowning) events in another study [30].

The incidence rates of drowning across the different studies ranged from a low of 0.33/100,000 population to a high of 502/100,000 population [21,22,24,25,27,43,54]. However, the denominators for each study varied. Two studies were conducted using the total population in the country as the denominator [21,22], five studies were conducted within specific cities and towns and total population of the cities were used as denominators $[23,24,27,43,54]$, while one study investigated drowning across five cities [25].

Of the 30 studies reporting drowning as part of a wider study (Table 2), 25 studies reported drowning as part of external cause of death (injury) [20,28,29,31-36,40-42,44-49,51-53,55,57-59], while 5 studies investigated all causes of deaths and reported the rates of drowning $[37-39,45,56]$. The studies investigating external causes of deaths reported drowning deaths either as a proportion $[28,29,31-34,40-42,44,46,47,49,51,52,55,57,61]$ or as a rate $[20,35,36,48,53,58,59]$ of all external causes of deaths. The proportion of drowning deaths as part of external causes of death ranged from $0.2 \%$ to $75 \%[28,29,31-34,40-42,44,46,47,49,51,52,55,57,61]$ while the incidence rate ranged from $2 \cdot 1 / 100,000$ to $10 \cdot 2 / 100,000[35,36,48,58,59]$. One ecological study which investigated external causes of deaths across eight (8) countries reported drowning rates ranging from $0 / 1000$ person years to 0.48/1000 person years [20], while. Lett et al., reported that drowning rate account for 0.1/1000 people of all external cause of deaths [53]. In studies reporting drowning as a part of all causes of deaths, the proportion of drowning deaths ranged from $0.02 \%$ to $11 \%$ [37-39,45,56].

In terms of drowning rates by age demographics, the proportion of drowning among children and adolescents ranged from $2.5 \%$ to $19 \%$ [28,30,31,33,37-39,42,46,49,52], while the incidence of drowning ranged from 0.33 to 5.3 per 100,000 population [21,35]. The rates of drowning among adults ranged from 0.33 to 502 per 100,000 population $[22,24,25,27,36,48,54,59]$ and from 0 to 0.07 per 1000 person years [20,53]. In addition, the proportion of drowning among adults ranged from $0.019 \%$ to $80 \%[23,26,29,32,34,40,41,44,45,50,51,55-58,60,61]$.

\subsection{Potential Risk Factors}

In Table 3, only two studies reported risk factors [48,58]. One study identified risk factors associated with drowning [58] and the other study reported risk factors of a wider study [58]. The identified risk factors were being a fisherman [58], having fishing as source of income [58], being male [58] and older age with the odds of drowning increasing with increasing age from 2.0 to 8.9 [58]. Potential risk factors were identified in 26 studies based on the rates of drowning reported and these potential factors include age, gender, ethnicity, alcohol, access to bodies of water, age of boat and carrying capacity of the boat, weather and summer season. In 19 studies, age was identified as a potential risk factor [24-28,30-33,37,40,42,46,47,55,57-60], with 13 studies reporting higher rates of drowning among children and adolescents $[25,27,28,30-33,37,42,43,46,47,59]$. In addition, males were reported to have higher rates of drowning compared to females in 15 studies [24-28,35,36,40,43,45-47,57,58,60]. Furthermore, six studies reported the rates of drowning for different races [24,26,28,31,35,42]. However, race as a potential risk factor varied between the included studies with three studies classifying the race by age groups $[24,31,42]$. The other three studies reported the rates of drowning for all age groups without grouping them $[26,28,35]$. Among studies reporting drowning rates for race by age groups, children aged $<1$ year -5 years of white African ethnicity were most vulnerable to drowning compared to other races. By contrast, drowning was more prevalent in adolescents and adults of black African ethnicity and Asian ethnicity $[24,26,31,35,42]$, while one study reported a higher rate of drowning among whites of African ethnicity [28]. Other potential risk factors identified were alcohol [23,25,26], summer season $[24,26,28,42,43]$, boat age and overloading of the boat [64], stormy weather [64] and access to bodies of water $[24-26,31,43,48,54,55]$. 
Table 1. Summary of studies exclusively reporting drowning in Africa.

\begin{tabular}{|c|c|c|c|c|c|}
\hline Authors, Reference, Year & Country & Study Design & Year & Study Population & $\begin{array}{c}\text { Rates and Proportion of } \\
\text { Drowning }\end{array}$ \\
\hline Davis and Smith, 1982 [23] & South Africa (Cape Town) & $\begin{array}{l}\text { Descriptive cross-sectional } \\
\text { study }\end{array}$ & 1979-1981 (3 years) & $\begin{array}{c}1,500,000 \text { people (population in } \\
\text { Cape Town) }\end{array}$ & $\begin{array}{c}285(0.019 \%) \text { drowning } \\
\text { deaths } \S\end{array}$ \\
\hline Grainger 1985 [60] & Seychelles & $\begin{array}{c}\text { Descriptive cross-sectional } \\
\text { study }\end{array}$ & 1959-1978 (20 years) & 119 drowning deaths & $\begin{array}{c}5.95 \pm 2.2 \text { drownings per } \\
\text { year (mean drowning rate) }\end{array}$ \\
\hline Davis and Smith 1985 [24] & South Africa (Cape Town) & $\begin{array}{l}\text { Descriptive cross-sectional } \\
\text { study }\end{array}$ & 1980-1983 (4 years) & $\begin{array}{c}1,600,000 \text { people (population in } \\
\text { Cape town) }\end{array}$ & $\begin{array}{l}\text { Male: } 38.7 / 100,000 \\
\text { Female: 8.3/100,000 }\end{array}$ \\
\hline Meel BL, 2008 [27] & South Africa (Mthatha) & $\begin{array}{l}\text { Descriptive cross-sectional } \\
\text { study }\end{array}$ & 1993-2004 (12 years) & $\begin{array}{l}400,000 \text { people (population in } \\
\text { Mthatha) }\end{array}$ & $\begin{array}{l}\text { Mean drowning rate: } \\
7.1 / 100,000\end{array}$ \\
\hline $\begin{array}{l}\text { Seleye-Fubara et al., } \\
2012[50]\end{array}$ & $\begin{array}{c}\text { Nigeria } \\
\text { (Niger-delta region) }\end{array}$ & $\begin{array}{l}\text { Descriptive cross-sectional } \\
\text { study }\end{array}$ & 1998-2009 (12 years) & 85 drowning deaths & $\begin{array}{l}80 \% \text { were unintentional } \\
\text { drowning }\end{array}$ \\
\hline $\begin{array}{c}\text { Donson and Nickerk, } \\
2013[25]\end{array}$ & South Africa & $\begin{array}{l}\text { Descriptive cross-sectional } \\
\text { study }\end{array}$ & 2001-2005 (5 years) & $\begin{array}{c}\text { Total population in five cities } \\
\text { (Johannesburg, Durban, } \\
\text { Cape Town, Port Elizabeth } \\
\text { and Pretoria) }\end{array}$ & $2.1 / 100,000$ \\
\hline Joanknecht et al., 2015 [30] & South Africa: Cape Town & $\begin{array}{l}\text { Descriptive cross-sectional } \\
\text { study }\end{array}$ & 2007-2013 (6 years) & $\begin{array}{l}75 \text { children admitted for a } \\
\text { submersion incident (near } \\
\text { drowning and drowning) }\end{array}$ & $\begin{array}{l}10.7 \% \text { of the study } \\
\text { population drowned }\end{array}$ \\
\hline Lin et al., 2015 [22] & $\begin{array}{c}\text { Egypt } \\
\text { South Africa }\end{array}$ & Ecological study & $\begin{array}{l}2009-2011 \text { ( } 3 \text { years }) \\
2007-2009 \text { ( } 3 \text { years) }\end{array}$ & $\begin{array}{l}\text { Entire population in the country } \\
\text { Entire population in the country }\end{array}$ & $\begin{array}{l}1.5 / 100,000 \\
2.5 / 100,000\end{array}$ \\
\hline Morris et al., 2016 [26] & South Africa (Pretoria) & $\begin{array}{l}\text { Descriptive cross-sectional } \\
\text { study }\end{array}$ & $2002-2011$ & $\begin{array}{l}23,050 \text { registered deaths } \\
278 \text { deaths due to } \\
\text { external causes }\end{array}$ & $\begin{array}{l}1.2 \%(278) \text { of the deaths } \\
\text { were due to drowning }\end{array}$ \\
\hline $\begin{array}{l}\text { Kobusingye et al., } \\
2017[54]^{\ddagger}\end{array}$ & $\begin{array}{l}\text { Uganda } \\
\text { (Buikwe; Kampala; } \\
\text { Mukono; Wakiso) }\end{array}$ & $\begin{array}{l}\text { Mixed methods: } \\
\text { Quantitative-Cross-sectional }\end{array}$ & Not stated & $\begin{array}{l}2804 \text { people (population in the } \\
\text { community) }\end{array}$ & $502 / 100,000$ \\
\hline Wu et al., 2017 [21] & $\begin{array}{c}\text { Egypt } \\
\text { South Africa }\end{array}$ & Ecological study & 2000 and 2013 & $\begin{array}{l}\text { WHO world standard } \\
\text { population } \\
\text { WHO world standard } \\
\text { population }\end{array}$ & 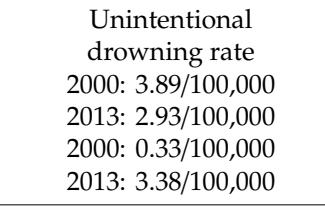 \\
\hline Saunders et al., 2018 [43] & $\begin{array}{l}\text { South Africa } \\
\text { (Western Cape) }\end{array}$ & Descriptive cross-sectional & 2010-2016 (6 years) & $\begin{array}{l}\text { Total population in Western } \\
\text { Cape (not stated by the authors) }\end{array}$ & $3.2 / 100,000$ \\
\hline
\end{tabular}

$\S$ Drowning proportions was calculated using data provided in the article. ${ }^{\ddagger}$ For the purpose of the review, only the quantitative aspect of the study was included in the review. 
Table 2. Studies describing drowning as part of other studies (including external causes and all causes) in Africa.

\begin{tabular}{|c|c|c|c|c|c|}
\hline Authors, Reference, Year & Country & Study Design & Year & Study Population & Rates and Proportion of Drowning \\
\hline Chitiyo 1974 [61] & $\begin{array}{c}\text { Zimbabwe } \\
\text { (Bulawayo area) }\end{array}$ & $\begin{array}{c}\text { Descriptive } \\
\text { cross-sectional study }\end{array}$ & 1972 & 188 adult deaths (external causes) & $11.17 \%$ (21) drowning deaths \\
\hline Knobel et al., 1984 [28] & South Africa & $\begin{array}{c}\text { Descriptive } \\
\text { cross-sectional study }\end{array}$ & 1966-1981 (15 years) & $\begin{array}{c}3248 \text { children }<15 \text { years (external } \\
\text { causes) }\end{array}$ & 356 drowning deaths $(11 \%)$ \\
\hline Kibel et al., 1990 [31] & South Africa & $\begin{array}{c}\text { Descriptive } \\
\text { cross-sectional study }\end{array}$ & 1981-1985 (5 years) & $\begin{array}{l}14,118 \text { children under } 15 \text { years of } \\
\text { age (deaths due to external causes) }\end{array}$ & $19 \%$ of all injury related deaths \\
\hline Flisher et al., 1992 [42] & South Africa & $\begin{array}{l}\text { Descriptive } \\
\text { cross-sectional study }\end{array}$ & 1984-1986 (3 years) & $\begin{array}{l}9288 \text { adolescent deaths due to } \\
\text { external causes }\end{array}$ & $10.8 \%$ of all deaths due to external causes \\
\hline Lerer et al., 1997 [32] & South Africa (Cape Town) & $\begin{array}{l}\text { Descriptive } \\
\text { cross-sectional }\end{array}$ & 1994 (1 year) & 3690 deaths due to external causes & $\begin{array}{l}2.6 \%(96) \text { of all non-natural mortality was } \\
\text { due to drowning }\end{array}$ \\
\hline $\begin{array}{l}\text { Kobusingye et al., } \\
2001[55]\end{array}$ & Uganda (Mukono district) & $\begin{array}{l}\text { Descriptive } \\
\text { cross-sectional }\end{array}$ & 1993-1998 (5 years) & 34 fatal injuries (external causes) & $\begin{array}{c}27 \% \text { (9) of fatal injuries were due to } \\
\text { drowning }\end{array}$ \\
\hline \multirow[t]{4}{*}{ Moshiro et al., 2001 [59] } & \multirow[t]{4}{*}{ Tanzania } & \multirow[t]{4}{*}{$\begin{array}{l}\text { Descriptive } \\
\text { cross-sectional study }\end{array}$} & \multirow[t]{4}{*}{ 1992-1998 (6 years) } & \multirow{4}{*}{$\begin{array}{c}\text { 64,756 persons in Dr es Salaam } \\
\text { 146,359 (population in Hai) } \\
\text { 103,053 (population in Morogoro) } \\
\text { 1478 deaths due to injuries } \\
\text { (external causes) all age groups }\end{array}$} & Overall drowning incidence not stated \\
\hline & & & & & $\begin{array}{c}\text { Female drowning rates/100,000 } \\
\text { Dar es Salaam: } 4.7\end{array}$ \\
\hline & & & & & Hai District: 5.5 \\
\hline & & & & & $\begin{array}{c}\text { Morogoro: } 5.1 \\
\text { Male drowning rates/ 100,000 } \\
\text { Dar es Salaam: } 9.2 \\
\text { Hai District: } 10.2 \\
\text { Morogoro: } 7.9\end{array}$ \\
\hline Lett et al., 2006 [53] & Uganda (Gulu district) & $\begin{array}{c}\text { Descriptive } \\
\text { cross-sectional study }\end{array}$ & 1994-1999 (5years) & $\begin{array}{l}8595 \text { people397 deaths due to } \\
\text { external causes }\end{array}$ & $0.1 / 1000$ people were due to drowning \\
\hline Osime et al., 2007 [51] & Nigeria (Benin City) & $\begin{array}{c}\text { Descriptive } \\
\text { cross-sectional study }\end{array}$ & 2001-2004 (4 years) & $\begin{array}{l}5446 \text { trauma related deaths } \\
\text { (external causes) }\end{array}$ & $\begin{array}{l}\text { Drowning accounted for } 0.8 \% \text { of all } \\
\text { trauma related deaths }\end{array}$ \\
\hline \multirow[t]{2}{*}{ Burrows et al., 2010 [35] } & \multirow[t]{2}{*}{ South Africa } & \multirow[t]{2}{*}{$\begin{array}{c}\text { Descriptive } \\
\text { cross-sectional study }\end{array}$} & \multirow[t]{2}{*}{ 2001-2003 (2 years) } & $\begin{array}{l}3,301,190 \text { children aged } 0-14 \text { years } \\
2923 \text { injury related deaths (external } \\
\text { causes) of children aged } 0-14 \text { years }\end{array}$ & Female: $2.1 / 100,000$ \\
\hline & & & & & Male: $5.3 / 100,000$ \\
\hline Ohene et al., 2010 [46] & Ghana (Accra) & $\begin{array}{l}\text { Descriptive } \\
\text { cross-sectional }\end{array}$ & 2001-203 (2 years) & $\begin{array}{l}151 \text { injury related deaths } \\
\text { (external causes) among } \\
\text { adolescents aged } 10-19 \text { years }\end{array}$ & $38 \%$ of deaths were due to drowning \\
\hline
\end{tabular}


Table 2. Cont.

\begin{tabular}{|c|c|c|c|c|c|}
\hline Authors, Reference, Year & Country & Study Design & Year & Study Population & Rates and Proportion of Drowning \\
\hline Garrib et al., 2011 [40] & South Africa & Analytical cross-sectional & 2000-2007 (7 years) & $\begin{array}{c}133,483 \text { people } \\
1022 \text { injury related deaths } \\
\text { (external causes) }\end{array}$ & $3.3 \%$ due to drowning \\
\hline Mendes et al., 2011 [29] & $\begin{array}{c}\text { South Africa } \\
\text { (Johannesburg) }\end{array}$ & $\begin{array}{c}\text { Descriptive } \\
\text { cross-sectional study }\end{array}$ & 2006-2009 (4 years) & $\begin{array}{c}1760 \text { unintentional injuries } \\
\text { (external causes) }\end{array}$ & $\begin{array}{l}0.34 \% \text { of the deaths were due to } \\
\text { drowning } \S\end{array}$ \\
\hline Mamady et al., 2012 [58] & Guinea & $\begin{array}{l}\text { Analytical cross-sectional } \\
\text { study }\end{array}$ & 2007 & $\begin{array}{c}\text { 9,710,144 (total population) } \\
7066 \text { fatal injuries (external causes) }\end{array}$ & $4.4 / 100,000$ \\
\hline Odhiambo et al., 2013 [57] & Kenya & Analytical cross-sectional & 2003-2008 (5 years) & $\begin{array}{l}\text { 220,000 people (total population) } \\
11,147 \text { adult deaths due to trauma } \\
\text { (external causes) }\end{array}$ & $0.2 \%$ (23) deaths were due to drowning \\
\hline $\begin{array}{c}\text { Weldearegawi et al., } \\
2013 \text { [45] }\end{array}$ & $\begin{array}{l}\text { Ethiopia (Kilite Awlaelo } \\
\quad \text { surveillance site) }\end{array}$ & $\begin{array}{c}\text { Descriptive } \\
\text { cross-sectional study }\end{array}$ & 2009-2011 (3 years) & 409 deaths (all causes) & $4.6 \%$ of all deaths were due to drowning \\
\hline Streatfield et al., 2014 [20] & $\begin{array}{c}\text { Burkina Faso } \\
\text { Cote d'Ivoire } \\
\text { Ethiopia } \\
\text { The Gambia } \\
\text { Ghana } \\
\text { Kenya } \\
\text { Malawi } \\
\text { South Africa }\end{array}$ & Ecological study & 2000-2012 (3 years) & $\begin{array}{l}111,910 \text { deaths/ } 12,204,043 \\
\text { person-years across Africa and Asia } \\
\text { due to external causes }\end{array}$ & $\begin{array}{c}\text { Rates/1000 person years } \\
\text { Burkina Faso (Nouna): } 0.00 \\
\text { Burkina Faso (Ouagadougou): } 0.20 \\
\text { Cote d'Ivoire (Taabo): } 0.14 \\
\text { Ethiopia (Kilite Awlaelo): } 0.13 \\
\text { The Gambia (Farafenni): } 0.11 \\
\text { Ghana (Dodowa): } 0.28 \\
\text { Ghana (Navrongo: } 0.48 \\
\text { Kenya (Kilifi): } 0.18 \\
\text { Kenya (Kisumu): } 0.22 \\
\text { Kenya (Nairobi): } 0.18 \\
\text { Malawi (Karonga): } 0.19 \\
\text { South Africa (Africa Centre): } 0.19 \\
\text { South Africa (Agincourt): } 0.07\end{array}$ \\
\hline $\begin{array}{l}\text { Chasimpha et al., } \\
2015 \text { [48] }\end{array}$ & Malawi (Karonga district) & Nested case-control & 20022012 & $\begin{array}{l}\text { 59,947 people (children and adults) } \\
\text { in Karonga districtDeaths due to } \\
\text { external causes }\end{array}$ & Unintentional drowning rate: $8.6 / 100,000$ \\
\hline Kone et al., 2015 [56] & Cote d'Ivoire & $\begin{array}{c}\text { Descriptive } \\
\text { cross-sectional study }\end{array}$ & 2009-2011 (3 years) & $\begin{array}{l}\text { 39,422 people (total population)712 } \\
\text { deaths (all causes) }\end{array}$ & $\begin{array}{c}\text { Unintentional drowning rates } \\
\text { Male * } \\
\text { 5-14: } 0.1 \% \\
\text { 15-49: } 0.3 \% \\
\text { Female * } \\
\text { 5-14 years: } 0.02 \%\end{array}$ \\
\hline
\end{tabular}


Table 2. Cont.

\begin{tabular}{|c|c|c|c|c|c|}
\hline Authors, Reference, Year & Country & Study Design & Year & Study Population & Rates and Proportion of Drowning \\
\hline $\begin{array}{l}\text { Matzopoulos et al., } \\
2015[36]\end{array}$ & South Africa & $\begin{array}{c}\text { Descriptive } \\
\text { cross-sectional study }\end{array}$ & 2009 & $\begin{array}{c}52,493 \text { injury related deaths } \\
\text { (external causes) }\end{array}$ & Unintentional drowning3.3/100,000 \\
\hline Olatunya et al., 2015 [52] & Nigeria (Ekiti State) & $\begin{array}{c}\text { Descriptive } \\
\text { cross-sectional study }\end{array}$ & 2012-2014 (2 years) & $\begin{array}{l}5264 \text { children admitted for injury } \\
\text { related incidents (external causes) }\end{array}$ & $\begin{array}{c}\text { Drowning accounted for } 4.54 \% \text { of } \\
\text { all injuries }\end{array}$ \\
\hline $\begin{array}{l}\text { Pretorius and Niekerk, } \\
2015 \text { [33] }\end{array}$ & South Africa: Guateng & $\begin{array}{l}\text { Descriptive } \\
\text { cross-sectional study }\end{array}$ & 2008-2011 (2 years) & $\begin{array}{c}\text { Total population in Gauteng } \\
5404 \text { fatal injuries (external causes) } \\
\text { in children aged 0-19 years }\end{array}$ & $\begin{array}{c}8.9 \% \text { of all fatal injuries were due to } \\
\text { drowning }\end{array}$ \\
\hline $\begin{array}{l}\text { Groenewald et al., } \\
2016 \text { [38] }\end{array}$ & $\begin{array}{l}\text { South Africa: } \\
\text { Western Cape }\end{array}$ & $\begin{array}{c}\text { Descriptive } \\
\text { cross-sectional study }\end{array}$ & 2011 & $\begin{array}{l}2412 \text { deaths (all causes) of children } \\
\text { under } 5 \text { years of age }\end{array}$ & $\begin{array}{l}\text { Drowning accounted for } 2.8 \% \text { of } \\
\text { all deaths }\end{array}$ \\
\hline Mathews et al., 2016 [37] & $\begin{array}{c}\text { South Africa: } \\
\text { Western Cape and } \\
\text { KwaZulu-Natal }\end{array}$ & $\begin{array}{l}\text { Descriptive } \\
\text { cross-sectional study }\end{array}$ & 2014 & 711 child deaths (all causes) & $\begin{array}{l}\text { Drowning deaths accounted for } 2.5 \% \text { of } \\
\text { all deaths }\end{array}$ \\
\hline Reid et al., 2016 [39] & $\begin{array}{l}\text { South Africa: } \\
\text { Western Cape }\end{array}$ & $\begin{array}{l}\text { Descriptive } \\
\text { cross-sectional study }\end{array}$ & 2011 & $\begin{array}{c}\text { 180,814 children under } 5 \text { years of } \\
\text { age (total population) } \\
1051 \text { under-5 deaths (all causes) }\end{array}$ & $11 \%$ of all deaths were due to drowning \\
\hline Meel BL, 2017 [34] & South Africa & Descriptive epidemiology & 1996-2015 (20 years) & $\begin{array}{l}\text { 24, } 693 \text { deaths due to unnatural } \\
\text { (external) causes }\end{array}$ & $\begin{array}{c}5.1 \% \text { of unnatural deaths were due to } \\
\text { drowning }\end{array}$ \\
\hline Purcell et al., 2017 [49] & Malawi: Lilongwe & $\begin{array}{l}\text { Descriptive } \\
\text { cross-sectional study }\end{array}$ & 2008-2013 (6 years) & $\begin{array}{c}\text { 30,462 children with } \\
\text { traumatic injuries } \\
343 \text { deaths due to external causes }\end{array}$ & $\begin{array}{c}11.4 \% \text { of the deaths were due to } \\
\text { drowning }\end{array}$ \\
\hline Erasmus et al., 2018 [41] & South Africa & $\begin{array}{l}\text { Descriptive } \\
\text { cross-sectional study }\end{array}$ & 2010-2014 (5 years) & $\begin{array}{l}184 \text { injuries related (external causes) } \\
\text { deaths over the time period }\end{array}$ & $\begin{array}{l}75 \%(138) \text { of the deaths were due to } \\
\text { drowning }\end{array}$ \\
\hline Gelaye et al., 2018 [44] & Ethiopia & $\begin{array}{c}\text { Descriptive } \\
\text { cross-sectional study }\end{array}$ & 2009-2013 (5 years) & $\begin{array}{l}623 \text { injury related deaths } \\
\text { (external causes) }\end{array}$ & $21.8 \%$ (136) deaths were due to drowning \\
\hline Ossei et al., 2019 [47] & Ghana & $\begin{array}{c}\text { Descriptive } \\
\text { cross-sectional study }\end{array}$ & 2008-2016 (8 years) & $\begin{array}{l}1470 \text { unnatural deaths } \\
\text { (external causes) }\end{array}$ & $\begin{array}{l}7.14 \% \text { of the deaths were due to } \\
\text { drowning }\end{array}$ \\
\hline
\end{tabular}

$\S$ Drowning proportions were calculated using data provided in the articles. ${ }^{*}$ Other age groups reported no drowning deaths. 
Table 3. Studies discussing potential risk factors for drowning among all age groups in Africa.

\begin{tabular}{|c|c|c|c|c|c|}
\hline $\begin{array}{c}\text { Authors, } \\
\text { Reference, Year }\end{array}$ & Country & Study Population & \multicolumn{2}{|c|}{ Proportions or Rates of Potential Risk Factors } & Potential Risk Factors/Risk Factors Identified \\
\hline $\begin{array}{c}\text { Davis and Smith, } \\
1982 \text { [33] }\end{array}$ & $\begin{array}{l}\text { South Africa } \\
\text { (Cape Town) }\end{array}$ & $1,500,000$ people & & & Alcohol \\
\hline \multirow[t]{11}{*}{$\begin{array}{c}\text { Knobel et al., } \\
1984 \text { [28] }\end{array}$} & South Africa & $\begin{array}{l}3248 \text { children } \\
<15 \text { years }\end{array}$ & \multicolumn{2}{|c|}{ Race } & Race: whites \\
\hline & & & Coloured & $10.7 \%$ & \\
\hline & & & White & $16.1 \%$ & \\
\hline & & & Black & $7.9 \%$ & \\
\hline & & & \multicolumn{2}{|c|}{ Gender } & Male \\
\hline & & & Male & $11.7 \%$ & \\
\hline & & & Female & $9.7 \%$ & \\
\hline & & & \multicolumn{2}{|c|}{ Age } & \\
\hline & & & $<1$ year & $6.9 \%$ & Age: 6-14 \\
\hline & & & $1-5$ years & $12.8 \%$ & \\
\hline & & & 6-14 years & $20.3 \%$ & $\begin{array}{l}\text { Summer season } \\
\text { Weekends }\end{array}$ \\
\hline \multirow[t]{15}{*}{$\begin{array}{c}\text { Davis and Smith } \\
1985 \text { [24] }\end{array}$} & $\begin{array}{l}\text { South Africa } \\
\text { (Cape Town) }\end{array}$ & $1,600,000$ people & & & Race: Black race for adults $>30$ years \\
\hline & & & Black race & $32.3 / 100,000$ & White race for children $0-5$ years \\
\hline & & & Colored & $24.2 / 100,000$ & Male \\
\hline & & & White & $13.4 / 100,000$ & \\
\hline & & & \multicolumn{2}{|c|}{ Gender } & \\
\hline & & & Male & $38.7 / 100,000$ & \\
\hline & & & Female & $8.3 / 100,000$ & \\
\hline & & & \multicolumn{2}{|c|}{ Age } & Age: $21-30$ \\
\hline & & & $0-5$ years & $13.3 \%$ & \\
\hline & & & 6-10 years & $5.8 \%$ & \\
\hline & & & $11-15$ years & $4.9 \%$ & \\
\hline & & & $16-20$ years & $10.98 \%$ & \\
\hline & & & $21-30$ years & $25.14 \%$ & Summer season \\
\hline & & & $31-40$ years & $15.61 \%$ & Swimming pools \\
\hline & & & $>40$ years & $24.28 \%$ & Alcohol \\
\hline
\end{tabular}


Table 3. Cont.

\begin{tabular}{|c|c|c|c|c|c|}
\hline $\begin{array}{c}\text { Authors, } \\
\text { Reference, Year }\end{array}$ & Country & Study Population & Proportions or Rat & Factors & Potential Risk Factors/Risk Factors Identified \\
\hline \multirow[t]{12}{*}{ Grainger 1985 [60] } & Seychelles & 119 drowning deaths & \multicolumn{2}{|c|}{ Age } & Age: 40-49 years \\
\hline & & & $0-9$ years & $6.72 \%$ & Epilepsy \\
\hline & & & 10-19 years & $13.44 \%$ & Head injury \\
\hline & & & 20-29 years & $16.8 \%$ & Time of day: $12-2 \mathrm{pm}$ \\
\hline & & & $30-39$ years & $18.5 \%$ & \\
\hline & & & $40-49$ years & $21.8 \%$ & \\
\hline & & & 50-59 years & $12.6 \%$ & \\
\hline & & & $60-69$ years & $5.88 \%$ & \\
\hline & & & $70+$ years & $4.2 \%$ & \\
\hline & & & \multicolumn{2}{|c|}{ Gender } & \\
\hline & & & Male & 109 deaths & Male \\
\hline & & & Female & 10 deaths & \\
\hline \multirow{19}{*}{$\begin{array}{l}\text { Kibel et al., } \\
1990 \text { [31] }\end{array}$} & South Africa & $\begin{array}{l}14,118 \text { children under } \\
15 \text { years of age }\end{array}$ & \multicolumn{2}{|c|}{ Age } & \multirow{19}{*}{$\begin{array}{l}\text { White race for children }<1 \text { year to } 4 \text { years } \\
\text { Black race for children aged 5-14 years } \\
\text { Site: } \\
\text { Swimming pools for white children } \\
\text { Dams and rivers for older black children }\end{array}$} \\
\hline & & & <1year & $7.4 \%$ & \\
\hline & & & 1-4 years & $23.0 \%$ & \\
\hline & & & $5-14$ years & $20.1 \%$ & \\
\hline & & & Race & $<1$ year & \\
\hline & & & Blacks & $6.7 \%$ & \\
\hline & & & Whites & $9.5 \%$ & \\
\hline & & & Coloured & $8.3 \%$ & \\
\hline & & & Asians & $6.4 \%$ & \\
\hline & & & & $1-4$ years & \\
\hline & & & Blacks & $18.8 \%$ & \\
\hline & & & Whites & $42.7 \%$ & \\
\hline & & & Coloured & $22.1 \%$ & \\
\hline & & & Asians & $9.4 \%$ & \\
\hline & & & & 5-14 years & \\
\hline & & & Blacks & $21.9 \%$ & \\
\hline & & & Whites & $12.7 \%$ & \\
\hline & & & Coloured & $21.2 \%$ & \\
\hline & & & Asians & $9.4 \%$ & \\
\hline
\end{tabular}


Table 3. Cont

\begin{tabular}{|c|c|c|c|c|c|}
\hline $\begin{array}{c}\text { Authors, } \\
\text { Reference, Year }\end{array}$ & Country & Study Population & \multicolumn{2}{|c|}{ Proportions or Rates of Potential Risk Factors } & Potential Risk Factors/Risk Factors Identified \\
\hline \multirow[t]{10}{*}{$\begin{array}{l}\text { Flisher et al., } \\
1992 \text { [42] }\end{array}$} & \multirow[t]{10}{*}{ South Africa } & \multirow[t]{10}{*}{$\begin{array}{l}9288 \text { adolescent deaths } \\
\text { due to external causes }\end{array}$} & Race & 10-14 years & Age: $10-14$ years \\
\hline & & & Whites & $6.3 \%$ & Black race for adolescents $10-14$ years old \\
\hline & & & Coloured & $25.2 \%$ & Asian race for adolescents $15-19$ years old \\
\hline & & & Asians & $12.7 \%$ & Summer season \\
\hline & & & Black & $24.2 \%$ & \\
\hline & & & Race & $15-19$ years & \\
\hline & & & Whites & $4.2 \%$ & \\
\hline & & & Coloured & $9.7 \%$ & \\
\hline & & & Asians & $12.5 \%$ & \\
\hline & & & Black & $6.3 \%$ & \\
\hline \multirow[t]{9}{*}{$\begin{array}{l}\text { Lerer et al., } \\
1997[32]\end{array}$} & \multirow[t]{9}{*}{$\begin{array}{l}\text { South Africa } \\
\text { (Cape Town) }\end{array}$} & \multirow[t]{9}{*}{$\begin{array}{l}3690 \text { non-natural } \\
\text { mortality }\end{array}$} & Age & $\begin{array}{c}\text { Number of } \\
\text { drowning deaths }\end{array}$ & Age: $0-14$ years \\
\hline & & & $0-14$ years: & 35 & \\
\hline & & & 15-24 years: & 10 & \\
\hline & & & 25-34 years & 16 & \\
\hline & & & $35-44$ years & 16 & \\
\hline & & & $45-54$ years & 12 & \\
\hline & & & 55-64 years & 3 & \\
\hline & & & $65-74$ years & 1 & \\
\hline & & & $75+$ years & 3 & \\
\hline \multirow[t]{7}{*}{$\begin{array}{c}\text { Kobusingye et al., } \\
2001 \text { [55] }\end{array}$} & \multirow{7}{*}{$\begin{array}{c}\text { Uganda } \\
\text { (Mukono district) }\end{array}$} & \multirow[t]{7}{*}{34 fatal injuries } & Age & $\%$ of drowning & Age: $10-39$ years \\
\hline & & & $<10$ years & $0 \%$ & Extensive water surface \\
\hline & & & $10-19$ years & $18 \%$ & \\
\hline & & & 20-29 years & $18 \%$ & \\
\hline & & & 30-39 years & $18 \%$ & \\
\hline & & & $40-49$ years & $0 \%$ & \\
\hline & & & $>50$ years & $0 \%$ & \\
\hline
\end{tabular}


Table 3. Cont

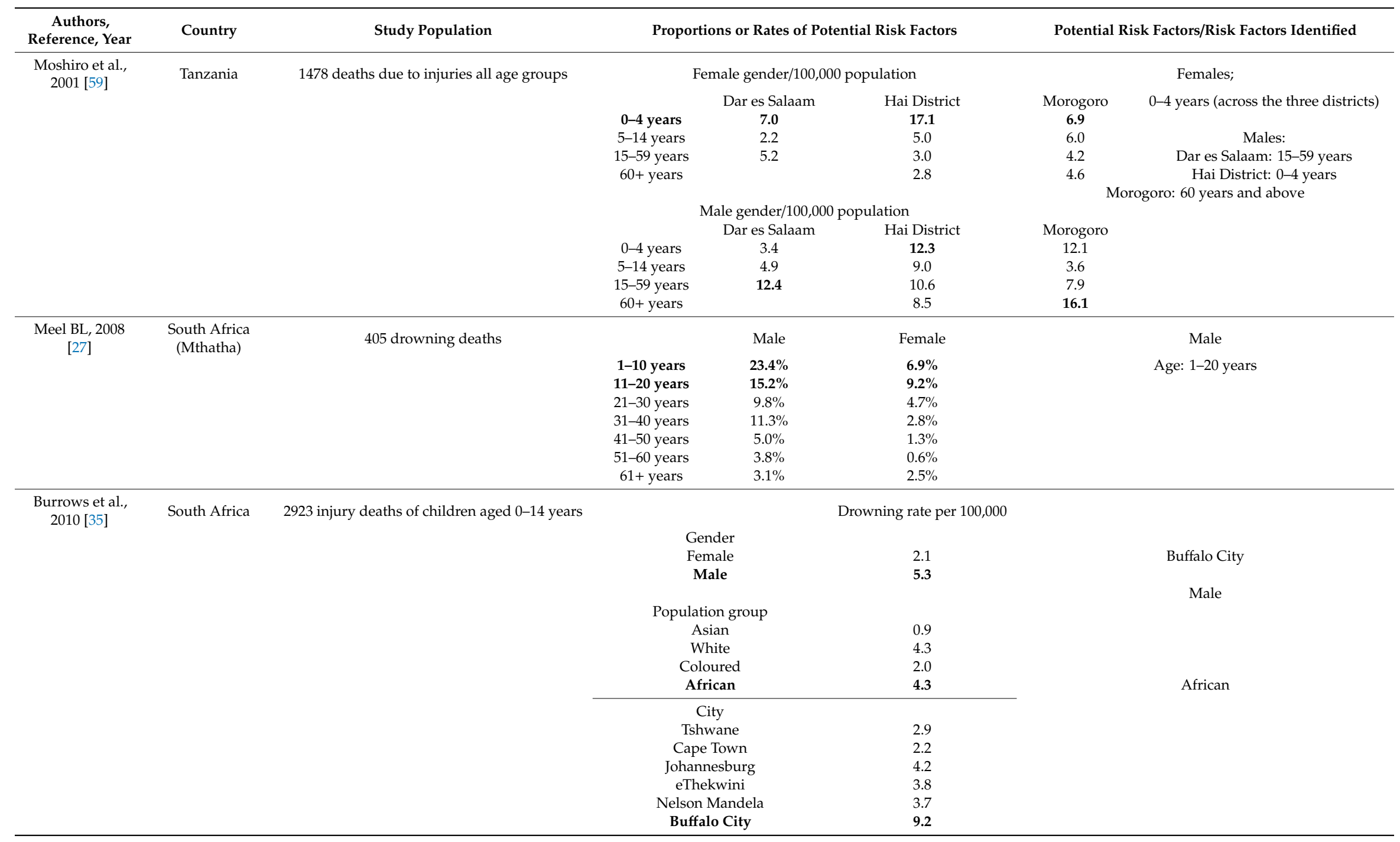


Table 3. Cont

\begin{tabular}{|c|c|c|c|c|c|}
\hline $\begin{array}{c}\text { Authors, } \\
\text { Reference, Year }\end{array}$ & Country & Study Population & Proportions or Rat & actors & Potential Risk Factors/Risk Factors Identified \\
\hline \multirow[t]{6}{*}{$\begin{array}{l}\text { Ohene et al., } \\
2010 \text { [46] }\end{array}$} & Ghana (Accra) & $\begin{array}{l}151 \text { injury related deaths among } \\
\text { adolescents aged } 10-19 \text { years }\end{array}$ & \multicolumn{2}{|c|}{ Gender } & Male \\
\hline & & & Female & $25 \%$ & \\
\hline & & & Male & $44 \%$ & \\
\hline & & & \multicolumn{2}{|c|}{ Age } & \multirow{3}{*}{ Age: $10-14$ years } \\
\hline & & & 10-14 years & $46 \%$ & \\
\hline & & & $15-19$ years & $33 \%$ & \\
\hline \multirow{6}{*}{$\begin{array}{l}\text { Garrib et al., } \\
2011 \text { [40] }\end{array}$} & South Africa & 1022 injury related deaths & \multicolumn{2}{|c|}{ Age } & \multirow[t]{4}{*}{ Children aged $0-15$ years } \\
\hline & & & $0-15$ years & $65 \%$ & \\
\hline & & & $>15$ years & $35 \%$ & \\
\hline & & & \multicolumn{2}{|c|}{ Gender (rate/100,000 person years) } & \\
\hline & & & Male & 6.2 & Male \\
\hline & & & Female & 3.4 & \\
\hline \multirow[t]{7}{*}{$\begin{array}{l}\text { Mamady et al., } \\
2012 \text { [58] }\end{array}$} & \multirow[t]{7}{*}{ Guinea } & \multirow[t]{7}{*}{7066 fatal injuries } & & & Female \\
\hline & & & & & OR $2.8 ; 95 \%$ CI (2.3-3.5) \\
\hline & & & & & $0-4$ years \\
\hline & & & & & OR 2.0; 95\% CI (1.1-3.5) \\
\hline & & & & & OR $8.9 ; 95 \%$ CI (5.3-15.0) \\
\hline & & & & & OR $7.0 ; 95 \%$ CI (4.2-11.7) \\
\hline & & & & & OR $7.9 ; 95 \%$ CI (4.4-14.3) \\
\hline \multirow[t]{2}{*}{$\begin{array}{l}\text { Seleye-Fubara } \\
\text { et al., } 2012 \text { [50] }\end{array}$} & \multirow[t]{2}{*}{$\begin{array}{c}\text { Nigeria } \\
\text { (Niger-delta region) }\end{array}$} & \multirow[t]{2}{*}{85 drowning deaths } & & & \multirow{2}{*}{$\begin{array}{l}\text { Alcohol } \\
\text { Hard drugs } \\
\text { Epilepsy }\end{array}$} \\
\hline & & & & & \\
\hline \multirow[t]{10}{*}{$\begin{array}{c}\text { Donson and } \\
\text { Nickerk, } 2013 \text { [25] }\end{array}$} & \multirow[t]{10}{*}{ South Africa } & \multirow[t]{10}{*}{1648 drowning deaths } & \multicolumn{2}{|c|}{ Age (rate/100,000) } & \\
\hline & & & $0-4$ years & 6.3 & \multirow{2}{*}{ 0-4-year age group } \\
\hline & & & $5-14$ years & 2.2 & \\
\hline & & & $15-29$ years & 1.7 & \multirow[t]{2}{*}{ Swimming pools } \\
\hline & & & $30-44$ years & 1.8 & \\
\hline & & & $45-59$ years & 1.4 & \multirow[t]{2}{*}{ Alcohol use } \\
\hline & & & $60+$ years & 1.2 & \\
\hline & & & Gende & & \multirow[t]{2}{*}{ December } \\
\hline & & & Male & 3.4 & \\
\hline & & & Female & 0.9 & Male \\
\hline
\end{tabular}


Table 3. Cont.

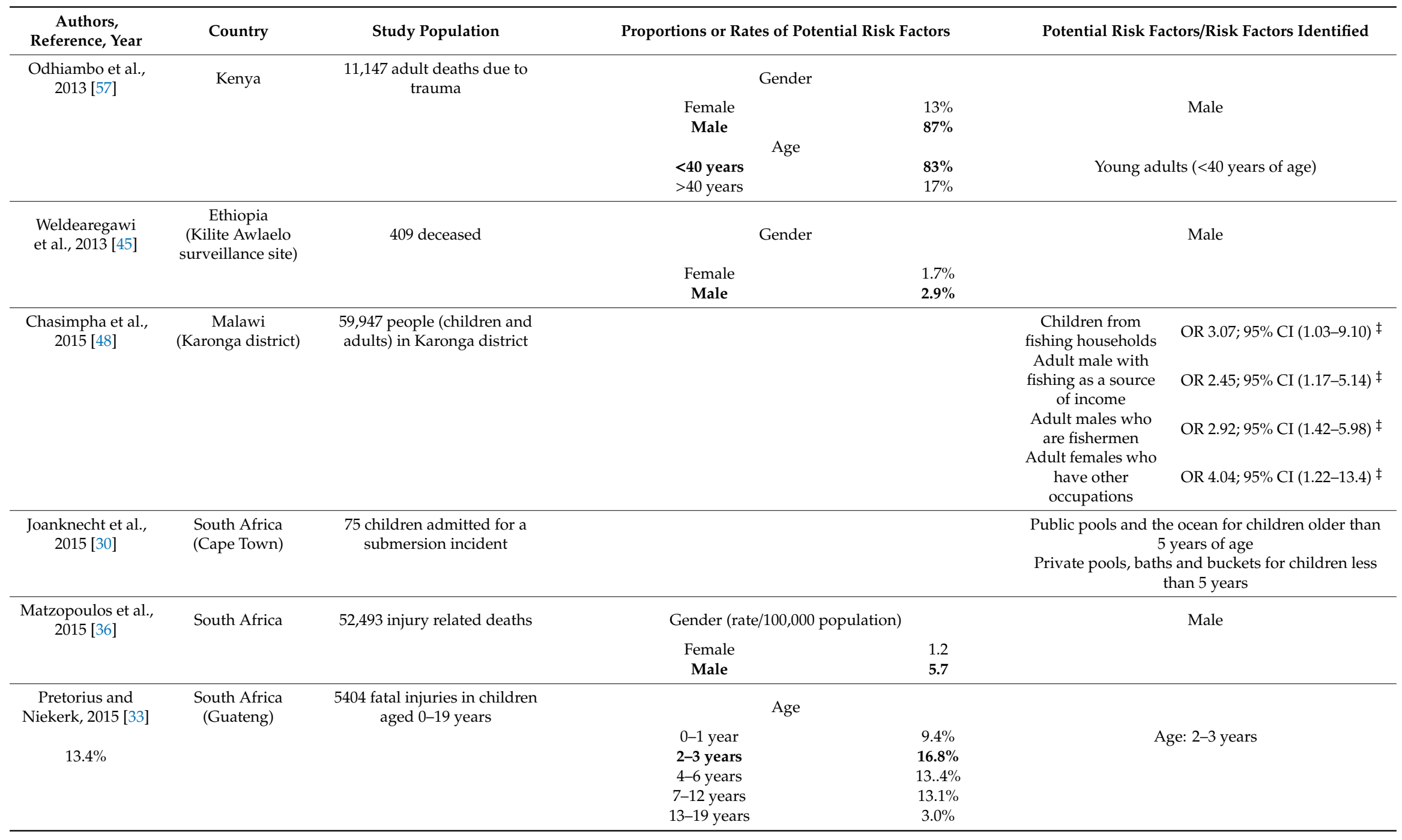


Table 3. Cont.

\begin{tabular}{|c|c|c|c|c|c|}
\hline $\begin{array}{c}\text { Authors, } \\
\text { Reference, Year }\end{array}$ & Country & Study Population & Proportions or Rat & actors & Potential Risk Factors/Risk Factors Identified \\
\hline \multirow[t]{5}{*}{$\begin{array}{l}\text { Mathews et al., } \\
2016 \text { [37] }\end{array}$} & $\begin{array}{c}\text { South Africa } \\
\text { (Western Cape and } \\
\text { KwaZulu-Natal) }\end{array}$ & 711 child deaths & \multicolumn{2}{|c|}{ Age } & \\
\hline & & & $<1$ year & $1.1 \%$ & Age: $1-4$ years \\
\hline & & & 1-4 years & $5.8 \%$ & \\
\hline & & & $5-14$ years & $5.0 \%$ & \\
\hline & & & $15-17$ years & $1.9 \%$ & \\
\hline \multirow[t]{14}{*}{$\begin{array}{l}\text { Morris et al., } \\
2016[26]\end{array}$} & $\begin{array}{l}\text { South Africa } \\
\text { (Pretoria) }\end{array}$ & $\begin{array}{l}346 \text { deaths due to external } \\
\text { causes }\end{array}$ & \multicolumn{2}{|c|}{ Gender } & \\
\hline & & & Female & $21 \%$ & Male \\
\hline & & & Male & $79 \%$ & \\
\hline & & & \multicolumn{2}{|c|}{ Race } & \\
\hline & & & Black & $71 \%$ & Black race \\
\hline & & & White & $24 \%$ & \\
\hline & & & Coloured & $4 \%$ & \\
\hline & & & Asian & $1 \%$ & \\
\hline & & & \multicolumn{2}{|c|}{ Age } & Age $>18$ years \\
\hline & & & $<1$ year & $15 \%$ & \\
\hline & & & $1-2$ years & $19 \%$ & Summer months (December to February) \\
\hline & & & $2-13$ years & $18 \%$ & Alcohol \\
\hline & & & $13-18$ years & $3 \%$ & Swimming pool \\
\hline & & & $>18$ years & $45 \%$ & \\
\hline \multirow[t]{4}{*}{$\begin{array}{l}\text { Kobusingye et al., } \\
\quad 2017 \text { [54] }\end{array}$} & $\begin{array}{l}\text { Uganda (Buikwe; } \\
\text { Kampala; Mukono; } \\
\text { Wakiso) }\end{array}$ & $\begin{array}{l}2804 \text { people (population in the } \\
\text { community) }\end{array}$ & & & Access to water bodies (for transportation or fishing) \\
\hline & & & & & Overloading \\
\hline & & & & & Stormy weather \\
\hline & & & & & Old age of boats \\
\hline \multirow[t]{3}{*}{ Meel BL, 2017 [34] } & South Africa & $\begin{array}{c}24,693 \text { deaths due to unnatural } \\
\text { causes }\end{array}$ & \multicolumn{2}{|c|}{ Gender } & Female \\
\hline & & & Female & $6.07 \%$ & \\
\hline & & & Male & $4.8 \%$ & \\
\hline \multirow{3}{*}{$\begin{array}{l}\text { Gelaye et al., } \\
2018[44]\end{array}$} & Ethiopia & 623 injury related deaths & \multicolumn{2}{|c|}{ Gender } & Female \\
\hline & & & Female & $22 \%$ & No formal education (illiterates) \\
\hline & & & Male & $21.1 \%$ & \\
\hline
\end{tabular}


Table 3. Cont.

\begin{tabular}{|c|c|c|c|c|c|}
\hline $\begin{array}{c}\text { Authors, } \\
\text { Reference, Year }\end{array}$ & Country & Study Population & Proportions or Rates of 1 & Factors & Potential Risk Factors/Risk Factors Identified \\
\hline \multirow[t]{6}{*}{$\begin{array}{l}\text { Saunders et al., } \\
\quad 2018[43]\end{array}$} & \multirow[t]{6}{*}{$\begin{array}{l}\text { South Africa } \\
\text { (Western Cape) }\end{array}$} & 1391 drowning deaths & \multicolumn{2}{|c|}{ Age (rate/100,000 population) } & Age: $0-19$ years \\
\hline & & & Children (0-19 years) & 3.8 & \\
\hline & & & Adults (20+ years) & 3.0 & \\
\hline & & & \multicolumn{2}{|c|}{ Gender (rate $/ 100,000$ population) } & \\
\hline & & & Female & 1.2 & Male \\
\hline & & & Male & 5.3 & $\begin{array}{l}\text { Access to large open bodies of water } \\
\text { Summer season (December, January, February) }\end{array}$ \\
\hline \multirow[t]{12}{*}{$\begin{array}{l}\text { Ossei et al., } \\
2019[47]\end{array}$} & \multirow[t]{12}{*}{ Ghana } & \multirow[t]{12}{*}{1470 unnatural deaths } & \multicolumn{2}{|c|}{ Age } & Age: $0-9$ years \\
\hline & & & $\leq 9$ years & $40 \%$ & \\
\hline & & & $10-19$ years & $17.14 \%$ & \\
\hline & & & $20-29$ years & $17.14 \%$ & \\
\hline & & & $30-39$ years & $10.48 \%$ & \\
\hline & & & $40-49$ years & $6.67 \%$ & \\
\hline & & & $50-59$ years & $3.81 \%$ & \\
\hline & & & $60-69$ years & $2.86 \%$ & \\
\hline & & & $\geq 70$ years & $1.90 \%$ & \\
\hline & & & \multicolumn{2}{|c|}{ Gender } & Male \\
\hline & & & Female & $22.9 \%$ & \\
\hline & & & Male & $77.1 \%$ & \\
\hline
\end{tabular}

$\ddagger$ Risk factors for external death, which also applies to drowning. 


\subsection{Prevention Strategies}

Sixteen (16) studies proposed prevention strategies to reduce drowning rates in Africa [23-27, $30,31,42,44-46,48,53,54,59,60]$. These prevention strategies include increased supervision of children around bodies of water, aquatic education and training about basic life support measures, training about life skills in communities, community awareness and implementation of legislation to prevent drowning (Table 4). Using the hierarchy of controls [64], fourteen of the sixteen studies proposed administrative control/preventive measures, which included education/training on basic life support, legislative laws and increasing public awareness $[23,25,27,30,31,42,44-46,48,53,54,59,60]$. Two studies proposed engineering control measure that include building life safety facilities and the use of barriers and safety nets around swimming pools $[24,26]$.

\subsection{Methodological Quality Assessment}

The QATSDD scores ranged from 53\% to 100\% (Supplementary Table S1). Thirty-three studies which scored above $80 \%$ [20-22,25,26,29-41,43-49,52-59], were categorised as having excellent methodological quality, and included details about sampling, data analysis, strengths and limitations of the study. The other studies were considered to be of good methodological quality $[23,24,27,28,42,50,51,60,61]$ and no study scored below $50 \%$. The included studies utilised retrospective data or collected information retrospectively from participants. Inaccuracy and incompleteness of the data may be associated with the use of retrospective data. In addition, misclassification bias may have been introduced into the studies that used retrospective data. Furthermore, depending on the participants, recall bias may have been introduced into some of the studies. These biases could have underestimated or overestimated the burden of drowning in Africa. 
Table 4. Studies discussing proposed prevention strategies for drowning among all age groups in Africa.

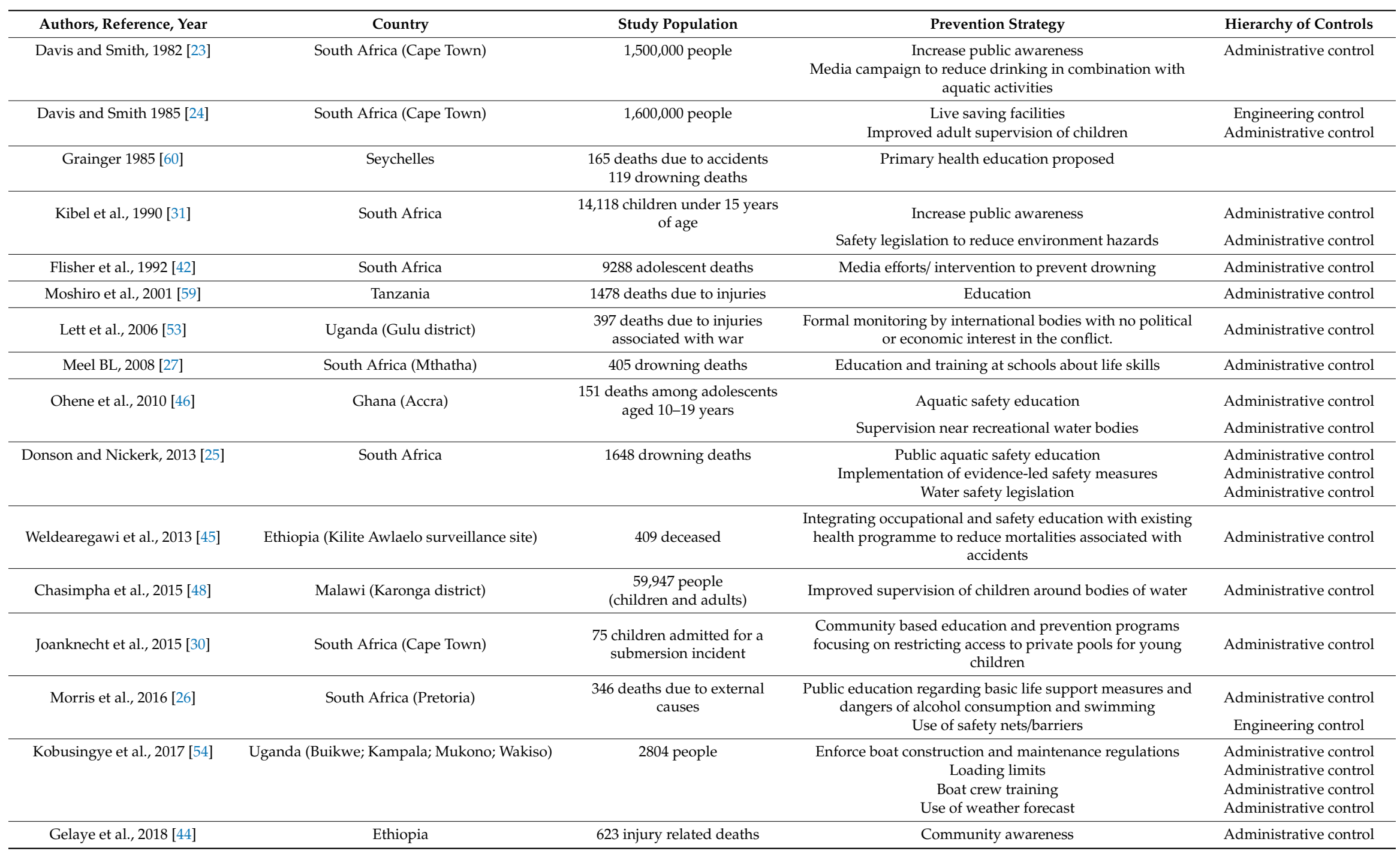




\section{Discussion}

Drowning is a significant public health burden in Africa and the findings of this systematic review suggest that there is a huge variation in drowning mortality across Africa. The highest proportion of drowning (approximately 80\%) was reported in Nigeria [50], while the highest rate reported (502/100,000 population) was observed in Uganda [54]. Although only two studies identified risk factors which includes being a fisherman, and older age [48,58]; we identified potential risk factors based on previous evidence [3]. The limited evidence suggests that male gender and young people are at higher risk for drowning especially children and adolescents. In addition, other potential risk factors identified were being of black African ethnicity, alcohol use, access to bodies of water, age of boat and carrying capacity of the boat, weather and summer season. This systematic review has highlighted the need for more data on drowning prevalence, together with good epidemiological studies across all African countries to describe the patterns of drowning and understand risk factors to guide prevention initiatives.

Due to the limited data available, quantifying the prevalence of drowning in Africa was challenging. This finding was echoed in a study of drowning in low- and middle-income countries by Tyler et al. who reported that inconsistences in data collection for drowning poses as a challenge for data synthesis [8]. Although the estimated rates and proportions may be considered high, in majority of the studies, drowning was reported as part of a wider injury study. Of the 54 countries in Africa, only 15 countries had some published data on drowning with the majority (57\%) of the literature originating from South Africa. In many African countries, cases go unreported and the lack of an injury surveillance system as seen in many LMIC also contributes to the limited data [65]. This is consistent with the findings of two recent systematic reviews describing the burden of drowning in South Africa and Tanzania [16,17]. Saunders et al. and Sarrassat et al., reported that strengthening the existing surveillance systems or establishing new ones are needed for consistent and detailed drowning surveillance $[16,17]$. In addition, as many of the drowning cases result in death at the time of the event, only a small proportion present at the hospital or medical facilities [66]. Both the lack of the injury surveillance system and underreporting of drowning cases prevent accurate documentation of drowning mortality in health records. According to WHO, approximately $90 \%$ of global drowning deaths occur in LMICs. Africa as a region had an estimated 73,635 drowning deaths in 2016 which accounted for approximately $23 \%$ of the total drowning deaths globally [67]. However, data collection in the region is limited, and hence the statistics from Africa underrepresents the true burden of drowning in the region [4].

Many of the potential risk factors associated with drowning identified in this review are similar to those reported in previous systematic reviews. Drowning occurred more frequently in males between the ages of 0 to 15 years. Specifically, highest drowning occurrences were found in the $0-5$-year age group. In LMICs, drowning rates among children were higher among children aged 1-4 years, followed by children aged 5-9 years with males being twice as likely as females to drown [8]. In addition, younger children were found to drown in private pools or baths, whereas older children were found to drown in public swimming pools, rivers, dams or in the ocean. Given that a majority of the studies originated from South Africa, drowning in swimming pools occurred more in white African children, whereas drowning in dams and rivers were found to occur in older black African children [24,31]. Although it is not evident that socioeconomic status is a potential risk factor in this review, children from low-income households may not have access to private swimming pools and are more likely to access natural bodies of water around the house as reported in other LMICs [8]. Evidence suggests that a lack of child supervision and the lack of safety barriers has been associated with high drowning rates among children [66]. Furthermore, access to other water bodies using boats or through fishing, depending on occupational or recreational purposes was also considered as a potential risk factor $[48,54]$. Specifically, children and adults from fishing households, are more likely to access these types of water bodies daily, requiring significant surveillance and awareness strategies for children in such settings. Other potential risk factors such as alcohol consumption has been shown to be associated 
with drowning especially among adolescents and adult [68]. As blood alcohol concentration level rises, judgement, balance and vision may be impaired, increasing the risk of drowning. Binge drinking is common in some African countries and has been reported to be associated with drowning among adult men [23-26,50]. This calls for increased awareness of the risk of alcohol consumption in conjunction with swimming.

The prevention strategies proposed by sixteen studies includes focusing on pool safety such as restricting access to private pools for young children, education and training at schools on life skills, increasing public awareness through media campaigns, and the implementation of water safety legislation, community awareness, improved supervision of children around water bodies, building lifesaving facilities and enforcement of boat construction and maintenance regulations. Using the hierarchy of controls which is a system used to minimize or eliminate hazards [64], only two studies proposed engineering controls as a way of preventing drowning [24,26]. All other studies proposed prevention strategies that require administrative controls which is the least effective way to prevent drowning $[23,25,27,30,31,42,44-46,48,53,54,59,60]$. However, prevention interventions and methods may not be consistent between countries due to the diversity and variation in their epidemiology, demographic and cultural characteristics [8]. There is no simple solution to addressing the burden of drowning in all countries, therefore strategies would have to be designed specifically for each country, keeping in mind the cultural, economic and social structures.

\subsection{Implications for Policy and Future Research}

The findings of this review suggest that there is limited evidence and data on the burden of drowning in Africa. The 2017 Global Burden of Disease (GBD) using statistical models estimated that drowning contributed approximately $0.53 \%$ of the total deaths in Africa as a region. Using the GBD to obtain country specific estimates for the countries included in the review showed an average drowning mortality ranging from 1.63 per 100,000 population to 5.73 per 100,000 population [69]. However, existing data on drowning in many countries in Africa are scarce. Therefore, establishing specific databases about injuries like drowning for surveillance and data collection would aid in development of policies and prevention strategies across the different countries. Evidence from research conducted in high income countries like Australia, Canada and New Zealand suggest that robust high-quality data and better data collection system would enable the creation of targeted and effective drowning prevention interventions [70]. Developing the databases will enable cross-country comparison which allows for identification of similarities and improvements in data collection. However, establishing the databases may be challenging for some African countries, especially if drowning is not among the national health priorities. In addition, there was little exploration of the risk factors associated with drowning, highlighting a gap in the literature. Good epidemiological studies are needed to identify the risk factors and evaluate the proposed prevention strategies for drowning in Africa. Furthermore, future research should focus on the intent for drowning in Africa, which would help to inform policies and prevention interventions. A recent article on intentional drowning reported an increasing rate of intentional drowning and proposed a multidisciplinary collaboration public health and other services including mental health, education and drowning prevention organisations to prevent intentional drowning [71].

\subsection{Strengths and Limitations}

To our knowledge, this is the first systematic review that describes the epidemiology, risk factors and prevention strategies of drowning in Africa. However, comparing mortality data across the countries within Africa needs to be undertaken with caution given the different measures used to analyse the burden of drowning. Some studies were population-based studies, while other studies reported drowning as a part of a wider study (such as external causes of deaths or all causes of death). An example of the latter is the study by Seleye-Fubara et al. which reported that unintentional drowning accounted for approximately $80 \%$ of all drowning deaths [50]. In addition, the completeness 
and reliability of the data in each country varied with some studies using the national mortality surveillance statistics, while other studies relied on hospital-based data, mortuary-based data and demographic surveillance data. The variability in the sources of data may account for the variable rates of drowning reported in the review.

Other limitations include reviewing only articles published in peer-reviewed journals. We may have missed other high-quality studies that are published in non-peer reviewed journals. In addition, we excluded non-English articles, there is a possibility that we may have missed articles from Africa published in other languages. Furthermore, the majority of studies were from South Africa, it is conspicuous that there is a paucity of data from many countries in Africa. It is uncertain whether such strategies, if implemented, can be generalizable to other African countries besides South Africa.

\section{Conclusions}

There is a need to address the high rate of drowning in Africa. It is imperative that governments across the nations of Africa establish good injury surveillance systems to accurately understand the burden of drowning to inform approaches for drowning prevention. Good epidemiological studies across all African countries are needed to describe the patterns of drowning and understand risk factors. Further research is needed to investigate the risk factors and to evaluate prevention strategies.

Supplementary Materials: The following are available online at http://www.mdpi.com/1010-660X/55/10/637/s1. Table S1: Medline search strategy; Table S2: Quality assessment of included studies using the quality assessment tool for studies with diverse designs (QATSDD); Figure S1: Schematic of study inclusion.

Author Contributions: All authors contributed equally to the study concept, design, data extraction, quality assessment and writing of the paper. All authors read and approved the manuscript for submission.

Funding: This research received no external funding.

Conflicts of Interest: The authors declare that the research was conducted in the absence of any commercial or financial relationships that could be construed as a potential conflict of interest.

\section{References}

1. van Beeck, E.F.; Branche, C.; Szpilman, D.; Modell, J.H.; Bierens, J.J. A new definition of drowning: Towards documentation and prevention of a global public health problem. Bull. WHO 2005, 83, 853-856. [PubMed]

2. World Health Organization (WHO). Drowning: 2019. Available online: https://www.who.int/violence_ injury_prevention/drowning/en/ (accessed on 17 June 2019).

3. World Health Organization (WHO). Drowning: 2018. Available online: https://www.who.int/en/news-room/ fact-sheets/detail/drowning (accessed on 17 June 2019).

4. World Health Organization. Global Report on Drowning: Preventing a Leading Killer; World Health Organization: Geneva, Switzerland, 2014.

5. Haagsma, J.A.; Graetz, N.; Bolliger, I.; Naghavi, M.; Higashi, H.; Mullany, E.C.; Abera, S.F.; Abraham, J.P.; Adofo, K.; Alsharif, U.; et al. The global burden of injury: Incidence, mortality, disability-adjusted life years and time trends from the Global Burden of Disease study 2013. Inj. Prev. 2016, 22, 3-18. [CrossRef] [PubMed]

6. World Health Organization (WHO). The African Regional Health Report: The Health of the People; World Health Organization (WHO): Geneva, Switzerland, 2014; p. 214.

7. Agbo, N. D’Banj Loses Son. The Guardian. 2018. Available online: https://guardian.ng/life/dbanj-loses-son/ (accessed on 15 May 2019).

8. Tyler, M.D.; Richards, D.B.; Reske-Nielsen, C.; Saghafi, O.; Morse, E.A.; Carey, R.; Jacquet, G.A. The epidemiology of drowning in low- and middle-income countries: A systematic review. BMC Public Health 2017, 17, 413. [CrossRef] [PubMed]

9. Borse, N.; Sleet, D.A. CDC Childhood Injury Report: Patterns of Unintentional Injuries Among 0- to 19-Year Olds in the United States, 2000-2006. Fam. Community Health 2009, 32, 189. [CrossRef] [PubMed]

10. Modell, J.H. Prevention of needless deaths from drowning. South. Med. J. 2010, 103, 650-653. [CrossRef] [PubMed]

11. Moran, K.; Quan, L.; Franklin, R.; Bennett, E. Where the evidence and expert opinion meet: A review of open-water recreational safety messages. Int. J. Aquat. Res. Educ. 2011, 5, 5. [CrossRef] 
12. Quan, L.; Bennett, E.E.; Branche, C.M. Interventions to prevent drowning. In Handbook of Injury and Violence Prevention; Doll, L., Bonzo, S., Sleet, D., Mercy, J., Haas, E.N., Eds.; Springer: Berlin/Heidelberg, Germany, 2008; pp. 81-96.

13. Szpilman, D.; Bierens, J.J.; Handley, A.J.; Orlowski, J.P. Drowning. N. Engl. J. Med. 2012, 366, $2102-2110$. [CrossRef] [PubMed]

14. Ramos, W.; Beale, A.; Chambers, P.; Dalke, S.; Fielding, R.; Kublick, L.; Langendorfer, S.J.; Lees, T.; Quan, L.; Wernicki, P. Primary and secondary drowning interventions: The American Red Cross circle of drowning prevention and chain of drowning survival. Int. J. Aquat. Res. Educ. 2015, 9, 8.

15. Bugeja, L.; Franklin, R.C. An analysis of stratagems to reduce drowning deaths of young children in private swimming pools and spas in Victoria, Australia. Int. J. Inj. Control Saf. Promot. 2013, 20, 282-294. [CrossRef]

16. Saunders, C.; Sewduth, D.; Naidoo, N. Keeping our heads above water: A systematic review of fatal drowning in South Africa. S. Afr. Med. J. 2018, 108, 61-68. [CrossRef] [PubMed]

17. Sarrassat, S.; Mrema, S.; Tani, K.; Mecrow, T.; Ryan, D.; Cousens, S. Estimating drowning mortality in Tanzania: A systematic review and meta-analysis of existing data sources. Inj. Prev. 2018. [CrossRef] [PubMed]

18. Moher, D.; Liberati, A.; Tetzlaff, J.; Altman, D.G. Preferred reporting items for systematic reviews and meta-analyses: The PRISMA statement. Ann. Intern. Med. 2009, 151, 264-269. [CrossRef] [PubMed]

19. Sirriyeh, R.; Lawton, R.; Gardner, P.; Armitage, G. Reviewing studies with diverse designs: The development and evaluation of a new tool. J. Eval. Clin. Pract. 2012, 18, 746-752. [CrossRef] [PubMed]

20. Streatfield, P.K.; Khan, W.A.; Bhuiya, A.; Hanifi, S.M.; Alam, N.; Diboulo, E.; Niamba, L.; Sié, A.; Lankoandé, B.; Millogo, R.; et al. Mortality from external causes in Africa and Asia: Evidence from INDEPTH Health and Demographic Surveillance System Sites. Glob. Health Action 2014, 7, 25366. [CrossRef] [PubMed]

21. Wu, Y.; Huang, Y.; Schwebel, D.C.; Hu, G.Q. Unintentional Child and Adolescent Drowning Mortality from 2000 to 2013 in 21 Countries: Analysis of the WHO Mortality Database. Int. J. Environ. Res. Public Health 2017, 14, 875. [CrossRef] [PubMed]

22. Ching-Yih, L.; Yi-Fong, W.; Tsung-Hsueh, L.; Ichiro, K. Unintentional drowning mortality, by age and body of water: An analysis of 60 countries. Inj. Prev. 2015, 21, e43-e50.

23. Davis, S.; Smith, L.S. Alcohol and drowning in Cape Town. A preliminary report. S. Afr. Med. J. 1982, 62, 931-933. [PubMed]

24. Davis, S.; Smith, L.S. The epidemiology of drowning in Cape Town-1980-1983. S. Afr. Med. J. 1985, 68, 739-742. [PubMed]

25. Donson, H.; Van Niekerk, A. Unintentional drowning in urban South Africa: A retrospective investigation, 2001-2005. Int. J. Inj. Control Saf. Promot. 2013, 20, 218-226. [CrossRef]

26. Morris, N.K.; Du Toit-Prinsloo, L.; Saayman, G. Drowning in Pretoria, South Africa: A 10-year review. J. Forensic Leg. Med. 2016, 37, 66-70. [CrossRef] [PubMed]

27. Meel, B.L.; Meel, B.L. Drowning deaths in Mthatha area of South Africa. Med. Sci. Law 2008, 48, 329-332. [CrossRef] [PubMed]

28. Knobel, G.J.; De Villiers, J.C.; Parry, C.D.H.; Botha, J.L. The causes of non-natural deaths in children over a 15-year period in greater Cape Town. S. Afr. Med. J. 1984, 66, 795-801. [PubMed]

29. Mendes, J.F.; Mathee, A.; Naicker, N.; Becker, P.; Naidoo, S. The prevalence of intentional and unintentional injuries in selected Johannesburg housing settlements. S. Afr. Med. J. 2011, 101, 835-838. [PubMed]

30. Joanknecht, L.; Argent, A.C.; van Dijk, M.; van As, A.B. Childhood drowning in South Africa: Local data should inform prevention strategies. Pediatr. Surg. Int. 2015, 31, 123-130. [CrossRef] [PubMed]

31. Kibel, S.M.; Joubert, G.; Bradshaw, D. Injury-related mortality in South African children, 1981-1985. S. Afr. Med. J. 1990, 78, 398-403. [PubMed]

32. Lerer, L.B.; Matzopoulos, R.G.; Phillips, R. Violence and injury mortality in the Cape Town metropole. S. Afr. Med. J. 1997, 87, 298-301. [PubMed]

33. Pretorius, K.; Van Niekerk, A. Childhood psychosocial development and fatal injuries in Gauteng, South Africa. Child Care Health Dev. 2015, 41, 35-44. [CrossRef] [PubMed]

34. Meel, B.L. Incidence of unnatural deaths in Transkei sub-region of South Africa (1996-2015). S. Afr. Fam. Pract. 2017, 59, 138-142. [CrossRef]

35. Burrows, S.; van Niekerk, A.; Laflamme, L. Fatal injuries among urban children in South Africa: Risk distribution and potential for reduction. Bull. World Health Organ. 2010, 88, 267-272. [CrossRef] [PubMed] 
36. Matzopoulos, R.; Prinsloo, M.; Pillay-van Wyk, V.; Gwebushe, N.; Mathews, S.; Martin, L.J.; Laubscher, R.; Abrahams, N.; Msemburi, W.; Lombard, C.; et al. Injury-related mortality in South Africa: A retrospective descriptive study of postmortem investigations. Bull. WHO 2015, 93, 303-313. [CrossRef] [PubMed]

37. Mathews, S.; Martin, L.J.; Coetzee, D.; Scott, C.; Naidoo, T.; Brijmohun, Y.; Quarrie, K. The South African child death review pilot: A multiagency approach to strengthen healthcare and protection for children. S. Afr. Med. J. 2016, 106, 895-899. [CrossRef] [PubMed]

38. Groenewald, P.; Bradshaw, D.; Neethling, I.; Martin, L.J.; Dempers, J.; Morden, E.; Zinyakatira, N.; Coetzee, D. Linking mortuary data improves vital statistics on cause of death of children under five years in the Western Cape Province of South Africa. Trop. Med. Int. Health 2016, 21, 114-121. [CrossRef] [PubMed]

39. Reid, A.E.; Hendricks, M.K.; Groenewald, P.; Bradshaw, D. Where do children die and what are the causes? Under-5 deaths in the Metro West geographical service area of the Western Cape, South Africa, 2011. S. Afr. Med. J. 2016, 106, 359-364. [CrossRef] [PubMed]

40. Garrib, A.; Herbst, A.J.; Hosegood, V.; Newell, M.L. Injury mortality in rural South Africa 2000 - 2007: Rates and associated factors. Trop. Med. Int. Health 2011, 16, 439-446. [CrossRef] [PubMed]

41. Erasmus, E.; Robertson, C.; van Hoving, D.J. The epidemiology of operations performed by the National Sea Rescue Institute of South Africa over a 5-year period. Int. Marit. Health 2018, 69, 1-7. [CrossRef] [PubMed]

42. Flisher, A.J.; Joubert, G.; Yach, D. Mortality from external causes in South African adolescents, 1984-1986. S. Afr. Med. J. 1992, 81, 77-80. [PubMed]

43. Saunders, C.J.; Adriaanse, R.; Simons, A.; van Niekerk, A. Fatal drowning in the Western Cape, South Africa: A 7-year retrospective, epidemiological study. Inj. Prev. 2018. [CrossRef] [PubMed]

44. Gelaye, K.A.; Tessema, F.; Tariku, B.; Abera, S.F.; Gebru, A.A.; Assefa, N.; Zelalem, D.; Dedefo, M.; Kondal, M.; Kote, M.; et al. Injury-related gaining momentum as external causes of deaths in Ethiopian health and demographic surveillance sites: Evidence from verbal autopsy study. Glob. Health Action 2018, 11. [CrossRef] [PubMed]

45. Weldearegawi, B.; Ashebir, Y.; Gebeye, E.; Gebregziabiher, T.; Yohannes, M.; Mussa, S.; Berhe, H.; Abebe, Z. Emerging chronic non-communicable diseases in rural communities of Northern Ethiopia: Evidence using population-based verbal autopsy method in Kilite Awlaelo surveillance site. Health Policy Plan. 2013, 28, 891-898. [CrossRef]

46. Ohene, S.A.; Tettey, Y.; Kumoji, R. Injury-related mortality among adolescents: Findings from a teaching hospital's post mortem data. BMC Res. Notes 2010, 3, 124. [CrossRef] [PubMed]

47. Ossei, P.P.S.; Ayibor, W.G.; Agagli, B.M.; Aninkora, O.K.; Fuseini, G.; Oduro-Manu, G.; Ka-Chungu, S. Profile of unnatural mortalities in Northern part of Ghana; a forensic-based autopsy study. J. Forensic Leg. Med. 2019, 65, 137-142. [CrossRef] [PubMed]

48. Chasimpha, S.; McLean, E.; Chihana, M.; Kachiwanda, L.; Koole, O.; Tafatatha, T.; Mvula, H.; Nyirenda, M.; Crampin, A.C.; Glynn, J.R. Patterns and risk factors for deaths from external causes in rural Malawi over 10 years: A prospective population-based study Health behavior, health promotion and society. BMC Public Health 2015, 15, 1036. [CrossRef] [PubMed]

49. Purcell, L.; Mabedi, C.E.; Gallaher, J.; Mjuweni, S.; McLean, S.; Cairns, B.; Charles, A. Variations in injury characteristics among paediatric patients following trauma: A retrospective descriptive analysis comparing pre-hospital and in-hospital deaths at Kamuzu Central Hospital, Lilongwe, Malawi. Malawi Med. J. 2017, 29, 146-150. [CrossRef] [PubMed]

50. Seleye-Fubara, D.; Nicholas, E.E.; Esse, I. Drowning in the Niger Delta region of Nigeria: An autopsy study of 85 cases. Niger. Postgrad. Med. J. 2012, 19, 111-114. [PubMed]

51. Osime, O.C.; Ighedosa, S.U.; Oludiran, O.O.; Iribhogbe, P.E.; Ehikhamenor, E.; Elusoji, S.O. Patterns of trauma deaths in an accident and emergency unit. Prehospital Disaster Med. 2007, 22, 75-78. [CrossRef] [PubMed]

52. Olatunya, O.S.; Isinkaye, A.O.; Oluwadiya, K.S. Profile of non-accidental childhood injury at a tertiary hospital in south-west Nigeria. J. Trop. Pediatr. 2015, 61, 174-181. [CrossRef] [PubMed]

53. Lett, R.R.; Kobusingye, O.C.; Ekwaru, P. Burden of injury during the complex political emergency in northern Uganda. Can. J. Surg. 2006, 49, 51-57.

54. Kobusingye, O.; Tumwesigye, N.M.; Magoola, J.; Atuyambe, L.; Olange, O. Drowning among the lakeside fishing communities in Uganda: Results of a community survey. Int. J. Inj. Control Saf. Promot. 2017, 24, 363-370. [CrossRef] 
55. Kobusingye, O.; Guwatudde, D.; Lett, R. Injury patterns in rural and urban Uganda. Inj. Prev. 2001, 7, 46-50. [CrossRef]

56. Koné, S.; Fürst, T.; Jaeger, F.N.; Esso, E.L.J.C.; Baïkoro, N.; Kouadio, K.A.; Adiossan, L.G.; Zouzou, F.; Boti, L.I.; Tanner, M.; et al. Causes of death in the Taabo health and demographic surveillance system, Cǒte d'Ivoire, from 2009 to 2011. Glob. Health Action 2015, 8, 27271. [CrossRef]

57. Odhiambo, F.O.; Beynon, C.M.; Ogwang, S.; Hamel, M.J.; Howland, O.; van Eijk, A.M.; Norton, R.; Amek, N.; Slutsker, L.; Laserson, K.F.; et al. Trauma-related mortality among adults in rural western Kenya: Characterizing deaths using data from a health and demographic surveillance system. PLoS ONE 2013, 8, e79840. [CrossRef] [PubMed]

58. Mamady, K.; Yao, H.; Zhang, X.; Xiang, H.; Tan, H.; Hu, G. The injury mortality burden in Guinea. BMC Public Health 2012, 12, 733. [CrossRef] [PubMed]

59. Moshiro, C.; Mswia, R.; Alberti, K.G.M.M.; Whiting, D.R.; Unwin, N.; Setel, P.W. The importance of injury as a cause of death in sub-Saharan Africa: Results of a community-based study in Tanzania. Public Health 2001, 115, 96-102. [CrossRef]

60. Grainger, C.R. Drowning accidents in the Seychelles. J. R. Soc. Health 1985, 105, 129-130. [CrossRef] [PubMed]

61. Chitiyo, M.E. Causes of unnatural adult deaths in the Bulawayo area. Cent. Afr. J. Med. 1974, 20, 57-60. [PubMed]

62. World Health Organization. ICD-11 for Mortality and Morbidity Statistics. Available online: https://icd.who. int/browse11/1-m/en\#/http\%3a\%2f\%2fid.who.int\%2ficd\%2fentity\%2f128104623 (accessed on 28 July 2019).

63. Wikimedia Commons. Blank Map of Africa. File: Blank Map-Africa.svg. Available online: https://commons. wikimedia.org/wiki/File:Blank_Map-Africa.svg (accessed on 20 May 2019).

64. Manuele, F.A. Risk assessment and hierarchies of control. Prof. Saf. 2005, 50, 33-39.

65. Cinnamon, J.; Schuurman, N. Injury surveillance in low-resource settings using Geospatial and Social Web technologies. Int. J. Health Geogr. 2010, 9, 25. [CrossRef] [PubMed]

66. Lukaszyk, C.; Ivers, R.Q.; Jagnoor, J. Systematic review of drowning in India: Assessment of burden and risk. Inj. Prev. 2018, 24, 451. [CrossRef]

67. World Health Organization. Global Health Estimates (GHE) 2016. Available online: https://www.who.int/ healthinfo/global_burden_disease/estimates/en/ (accessed on 13 June 2019).

68. Driscoll, T.R.; Harrison, J.A.; Steenkamp, M. Review of the role of alcohol in drowning associated with recreational aquatic activity. Inj. Prev. 2004, 10, 107. [CrossRef]

69. Institute for Health Metrics and Evaluation (IHME). GBD Compare Data Visualization. 2017. Available online: https://vizhub.healthdata.org/gbd-compare/ (accessed on 28 July 2019).

70. Peden, A.E.; Franklin, R.C.; Clemens, T. Exploring the burden of fatal drowning and data characteristics in three high income countries: Australia, Canada and New Zealand. BMC Public Health 2019, $19,794$. [CrossRef]

71. Cenderadewi, M.; Franklin, R.C.; Peden, A.E.; Devine, S. Pattern of intentional drowning mortality: A total population retrospective cohort study in Australia, 2006-2014. BMC Public Health 2019, 19, 207. [CrossRef] [PubMed]

(C) 2019 by the authors. Licensee MDPI, Basel, Switzerland. This article is an open access article distributed under the terms and conditions of the Creative Commons Attribution (CC BY) license (http://creativecommons.org/licenses/by/4.0/). 